\title{
Surgical treatment of double-lumen aortic arch (persistent fifth aortic arch) associated with severe aortic coarctation
}

\author{
Chang Hun Kim ${ }^{1}$, Hyungtae Kim ${ }^{1}$, Kwang Ho Choi ${ }^{1}$, Si Chan Sung ${ }^{1}$, and Hoon Ko ${ }^{1}$ \\ ${ }^{1}$ Pusan National University Yangsan Hospital
}

December 24, 2020

\begin{abstract}
Persistent fifth aortic arch (PFAA) is a rare anomaly often associated with aortic coarctation or interruption, and various surgical techniques for this anomaly have been reported. Herein, we show a case of an infant with PFAA and severe aortic coarctation. The aortic arch was reconstructed using end-to-side anastomosis between the fifth aortic arch and the descending aorta without any artificial conduit or patching material. This approach is easy and effective, has growth potential, and an additional surgery is not needed in the future.
\end{abstract}

\section{Introduction}

Persistent fifth aortic arch (PFAA) is a rare congenital cardiac malformation frequently associated with aortic coarctation or interruption ${ }^{1}$. Various surgical repair techniques for PFAA have been reported ${ }^{1-6}$. Herein, we show a case of an infant with PFAA and severe aortic coarctation, which was successfully repaired without any artificial conduits or patching material.

\section{Case report}

A 41-day-old female infant was admitted for sustained fever. The patient was diagnosed with bacterial meningitis by analysis of the cerebrospinal fluid and treated with intravenous antibiotics. During the hospital stay, the patient showed desaturation accompanied by cyanosis and cardiomegaly (cardiothoracic ratio, 0.77) on chest X-ray (Fig. 1A). Due to a constant increase in oxygen demand, the patient was intubated and transferred to the pediatric intensive care unit. Echocardiography showed coarctation of the aorta with decreased left ventricular function, atrial septal defect (ASD), and mitral valve regurgitation (Fig. 1B). Three-dimensional computed tomography (CT) angiography showed PFAA with aortic coarctation (Fig. 1C). Because the patient showed progressive oliguria and metabolic acidosis, we decided to perform emergency surgery.

The intraoperative findings revealed that the fourth aortic arch was connected to the descending aorta through a stenotic isthmic portion, and a stenotic area was also observed between the PFAA and the descending aorta (Fig. 2A). Cardiopulmonary bypass (CPB) was established by arterial cannulation of the innominate artery and bicaval venous cannulations. After cardiac arrest was induced, the connection between the fourth aortic arch and the descending aorta was divided. Antegrade cerebral perfusion was started under 25 of body temperature, and the CPB flow rate was $50 \mathrm{ml} / \mathrm{min} / \mathrm{kg}$. The PFAA was also divided, and the ductal tissue was resected completely from the descending aorta (Fig. 2B). The repair was performed in an end-to-side fashion between the proximal stump of the fifth aortic arch and the trimmed descending aorta (Fig. 2C). The ASD was closed primarily, and the CPB was weaned successfully. The perioperative period was uneventful. The patient was extubated three days after the operation. Postoperative echocardiography showed a wide aortic arch with improved left ventricular function and no mitral regurgitation. The 
postoperative CT showed a wide aortic arch as well (Fig. 3). The patient was discharged from the hospital 18 days after the operation and followed up 12 months without complications.

\section{Comment}

In 1969, Van Praagh and Van Praagh first reported a PFAA in a male patient ${ }^{7}$. PFAA is usually located below the fourth aortic arch. As a result, the distal aortic arch takes a bifurcated shape, which is also called a double-lumen aortic arch. Embryologically, mammals have six pairs of primitive pharyngeal arches, which form the aortic arch and the head and neck vessels. After normal development, the left fourth aortic arch develops as the normal left aortic arch, and both sixth aortic arches develop as the pulmonary arteries. Ductus arteriosus originates from the distal portion of the left sixth aortic arch. Both fifth aortic arches normally degenerate without leaving any remnants. However, when the fifth aortic arch persists and forms an additional aortic arch, it results in a double-lumen aortic arch without a known mechanism. In a review of the clinical practice of PFAA, PFAA was classified into four types according to the connection of the vessels and the direction of the blood flow: systemic-to-systemic, systemic-to-pulmonary, pulmonary-to-systemic, and bilateral types ${ }^{8}$. A review of 26 cases of PFAA by Lambert et al. ${ }^{1}$ found that $76 \%$ of the cases presented with systemic-to-systemic connections via the fifth aortic arch. Of them, $38 \%$ and $19 \%$ of the cases were associated with the aortic coarctation and interruption of the fourth aortic arch, respectively.

Various surgical methods for the PFAA with aortic coarctation have been reported previously including reconstruction of the fourth aortic arch using PFAA after ligation of the ductus arteriosus ${ }^{2}$, interposition of a Gore-Tex tube graft (W. L. Gore \& Assoc, Flagstaff, AZ, USA) between the ascending and descending aorta $^{6}$, patch aortoplasty augmenting both the fourth and fifth aortic arches ${ }^{6}$, side-to-side anastomosis of the left common carotid artery and left subclavian artery and patch augmentation of the coarcted segment ${ }^{5}$, end-to-end anastomosis of the PFAA and descending aorta after making one aortic arch from the fourth and fifth aortic arches ${ }^{1,3}$, and end-to-end anastomosis between the fifth aortic arch and the descending aorta ${ }^{4}$. In our case, the aortic arch was reconstructed using end-to-side anastomosis between the fifth aortic arch and the descending aorta after complete resection of the ductal tissue (Fig. 2). There were several advantages to our strategy. First, because the anastomosis is simply performed between the fifth aortic arch and the descending aorta, this method is easy and effective. Second, this technique can be performed using the patient's own tissue without any artificial patching material. Therefore, it has growth potential and no additional surgery is needed in the future.

In conclusion, PFAA with aortic coarctation is a rare congenital cardiovascular malformation, which can be repaired by various surgical methods. Among them, our surgical treatment option without the use of any artificial material showed good postoperative results and some benefits.

\section{Figures}

Figure 1. (A) Severe cardiomegaly was identified on the initial chest x-ray, and (B) preoperative echocardiography and (C) three-dimensional computed tomography angiography (posterior view) showed the persistent fifth aortic arch with severe aortic coarctation.

$\mathrm{DA}=$ descending aorta; $\mathrm{LA}=$ left atrium; PFAA $=$ persistent fifth aortic arch.

Figure 2. Schematic drawing of the operative technique. (A) The anatomic morphology of the aortic arch and (B) after division of the isthmic portion and the ductus arteriosus. (C) The proximal stump of the fifth aortic arch was anastomosed to the trimmed descending thoracic aorta in an end-to-side fashion.

Figure 3.Postoperative three-dimensional computed tomography showing a wide aortic arch without stenosis.

\section{References}

1. Lambert V, Blaysat G, Sidi D, Lacour-Gayet F. Double-lumen aortic arch by persistence of fifth aortic arch: A new case associated with coarctation. Pediatr Cardiol. 1999;20:167-9. 
2. Cabrera A, Galdeano J, Lekuona I. Persistent left sided fifth aortic arch in a neonate. Br Heart J. 1985;54:105-6.

3. Cetrano E, Polito A, Trezzi M, Carotti A. Neonatal Repair of Persistent Fifth Aortic Arch Coarctation and Interrupted Fourth Aortic Arch. Ann Thorac Surg. 2017;103:e475-e7.

4. Kim SH, Choi ES, Cho S, Kim WH. Persistent Fifth Aortic Arch with Coarctation. Korean J Thorac Cardiovasc Surg. 2016;49:39-41.

5. Yoshii S, Matsukawa T, Hosaka S, Ueno A, Tsuji A. Repair of coarctation with persistent fifth arterial arch and atresia of the fourth aortic arch. J Cardiovasc Surg (Torino). 1990;31:812-4.

6. Zhao YH, Su ZK, Liu JF, Cao DF, Ding WX. Surgical treatment of persistent fifth aortic arch associated with interrupted aortic arch.Ann Thorac Surg. 2007;84:1016-9.

7. Van Praagh R, Van Praagh S. Persistent fifth arterial arch in man. Congenital double-lumen aortic arch. Am J Cardiol.1969;24:279-82.

8. Lloyd DFA, Ho SY, Pushparajah K, Chakraborty S, Nasser M, Uemura H, et al. Persistent fifth aortic arch: the "great pretender" in clinical practice. Cardiol Young. 2018;28:175-81.

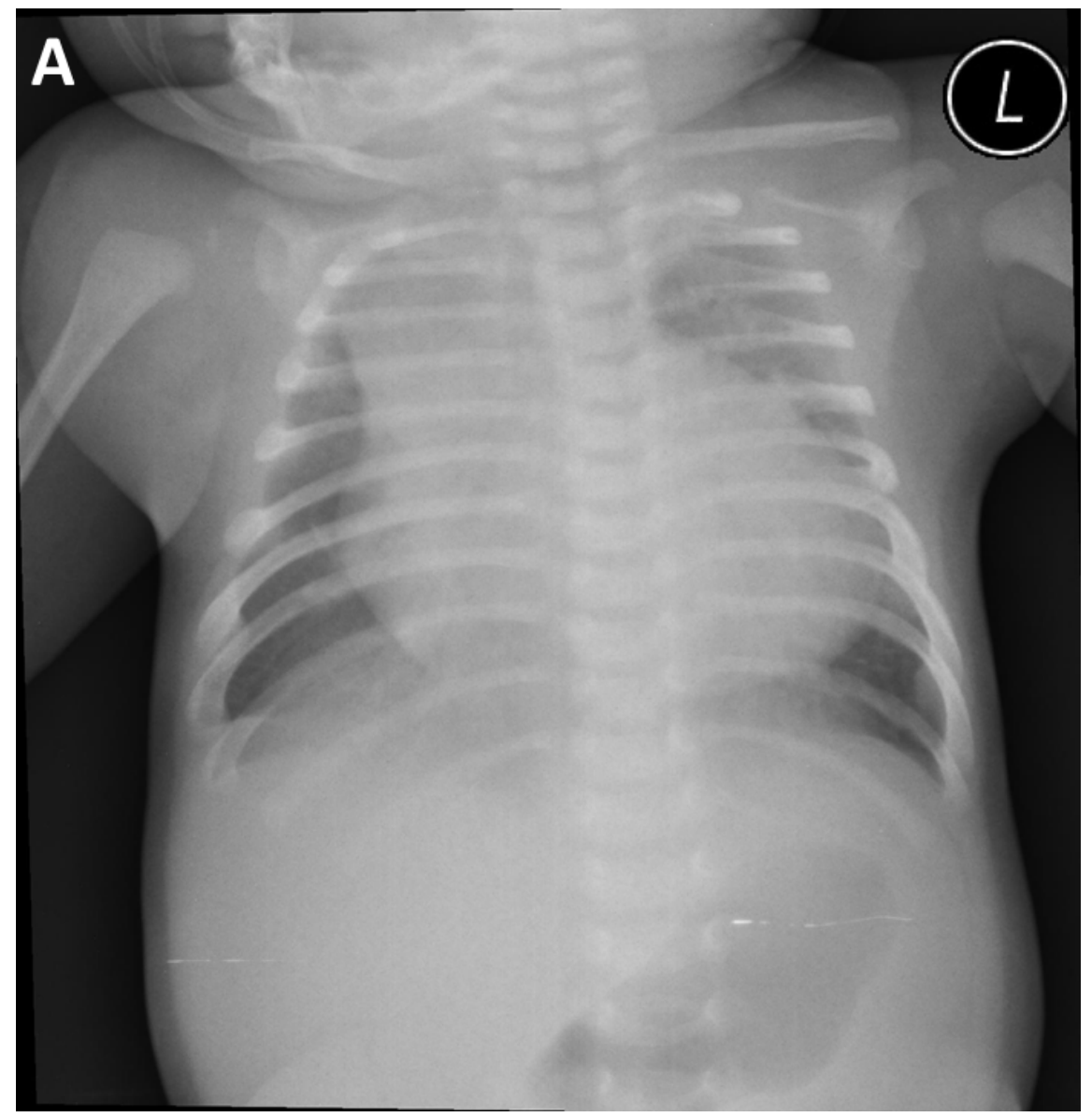




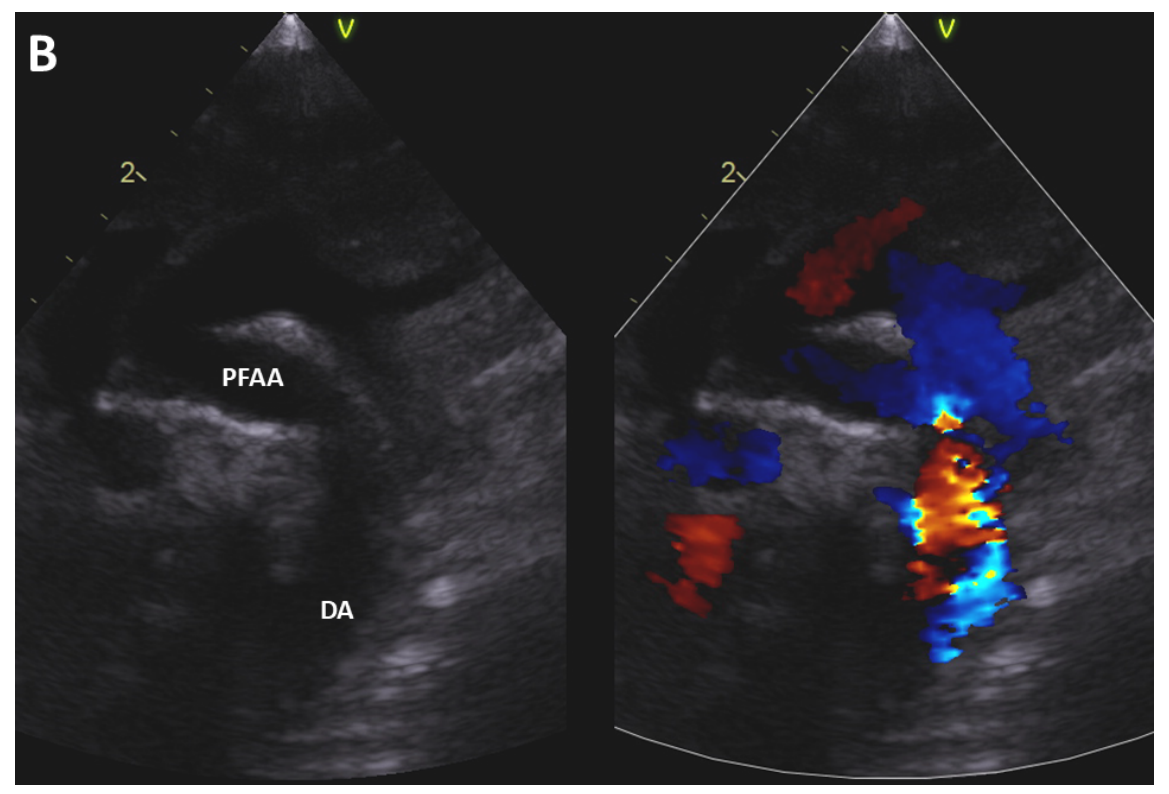




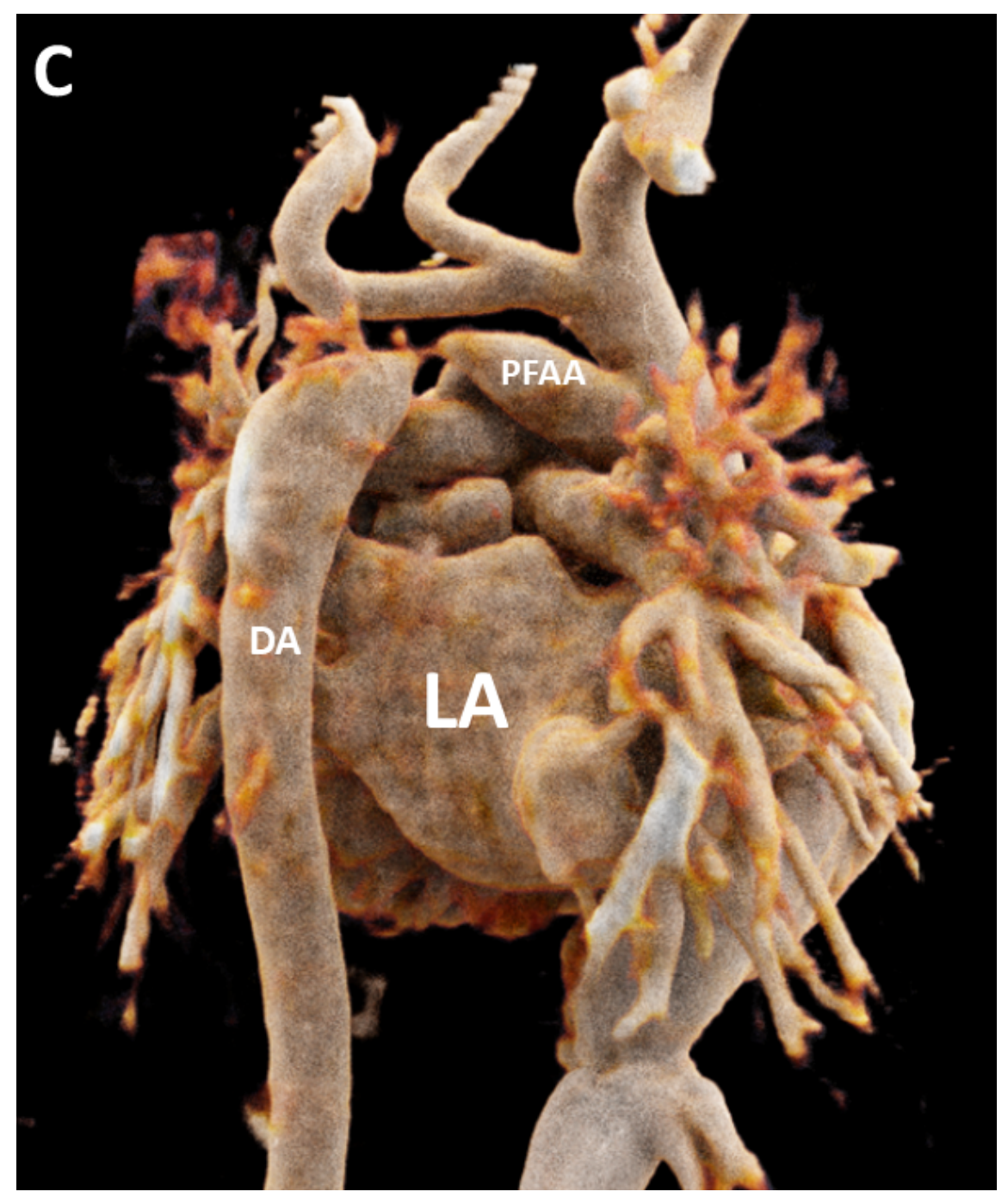




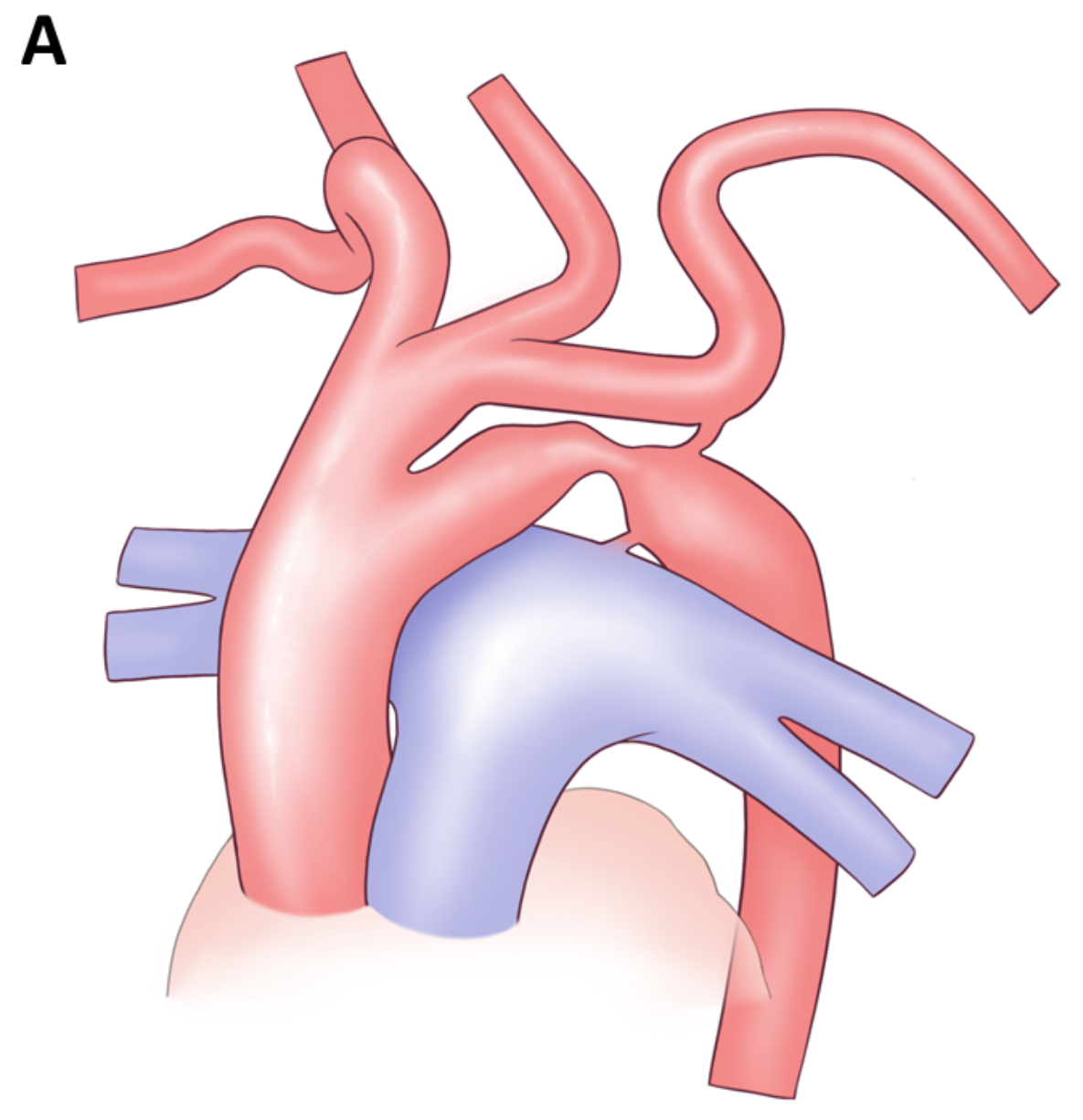




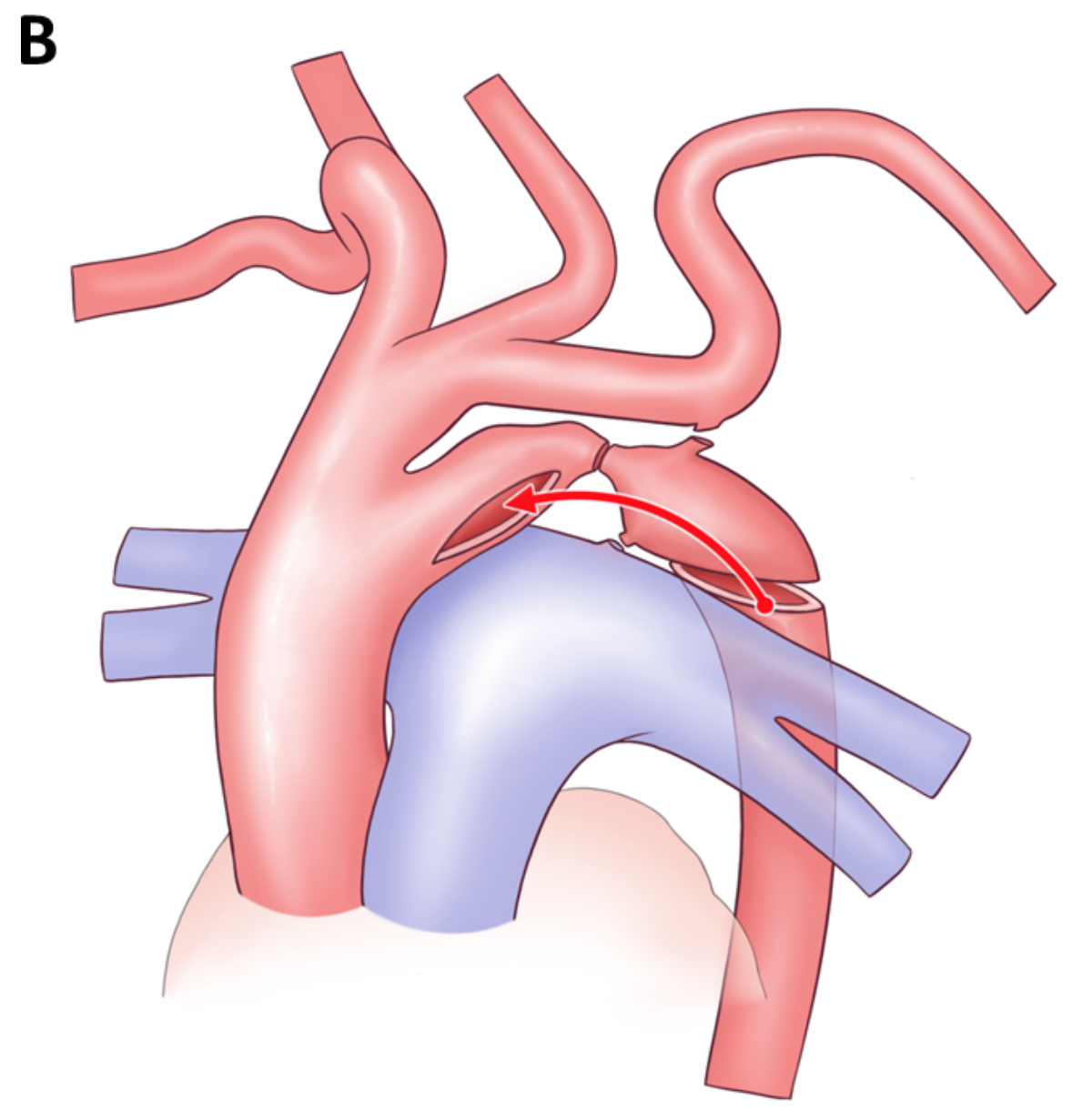




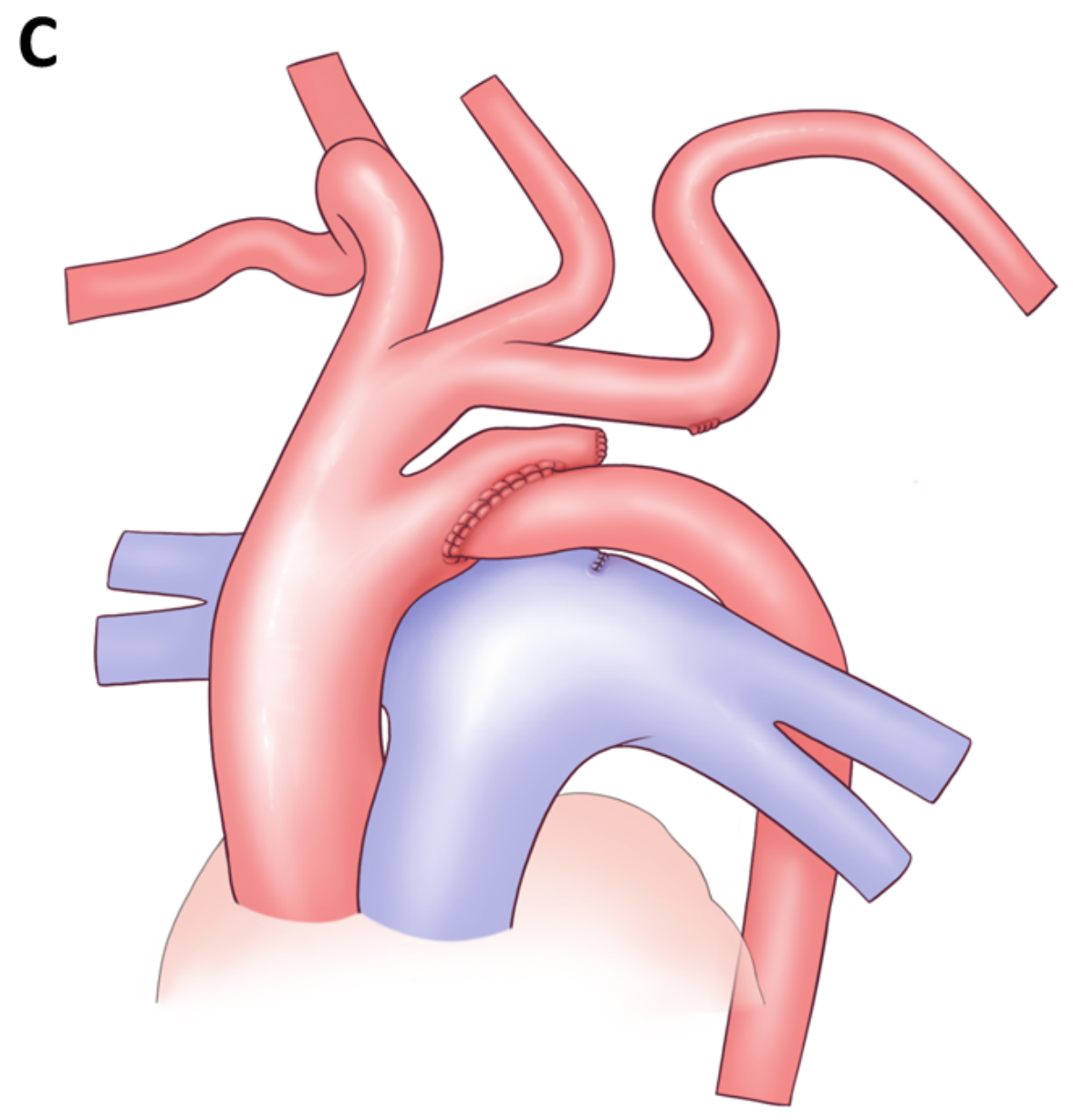




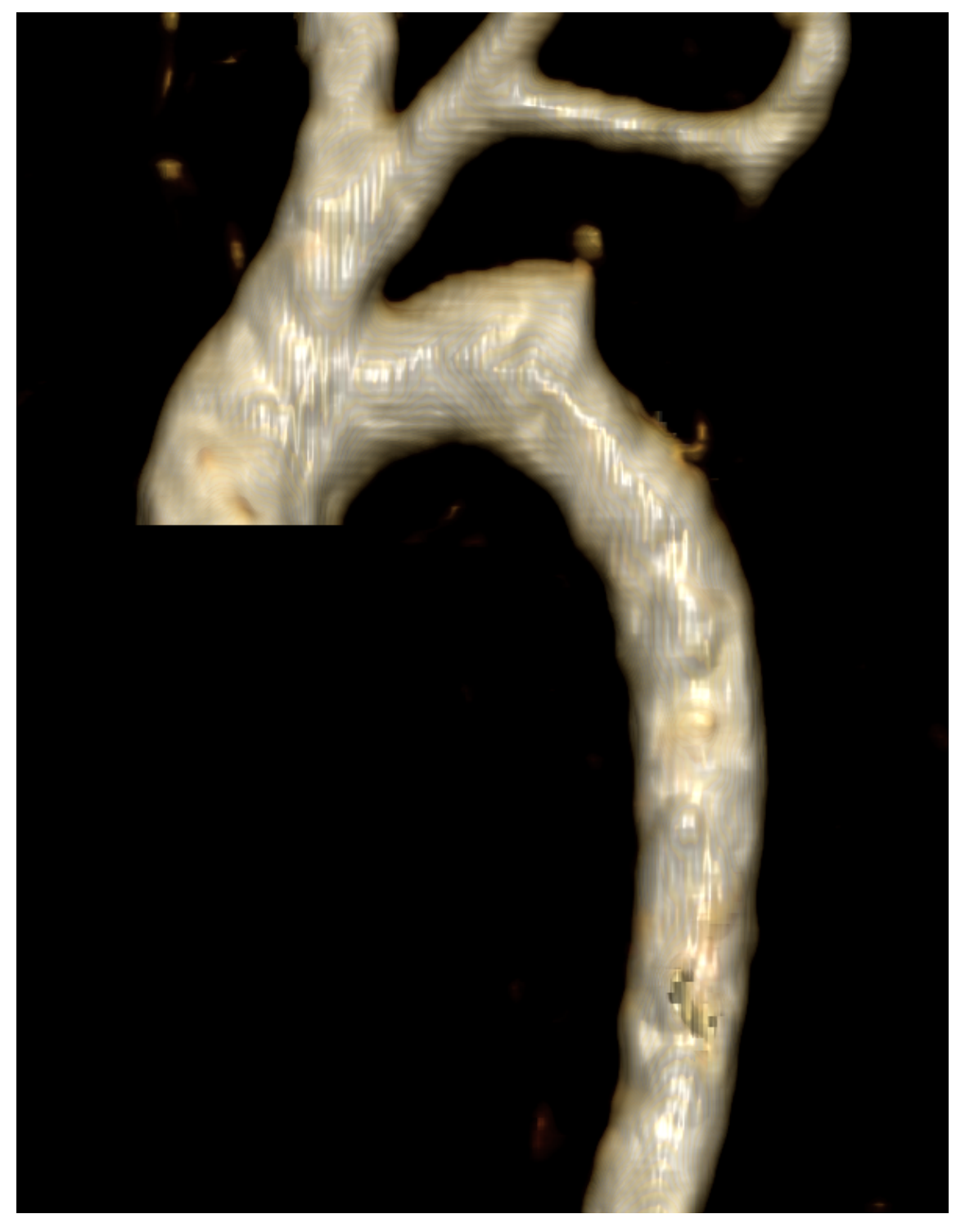

